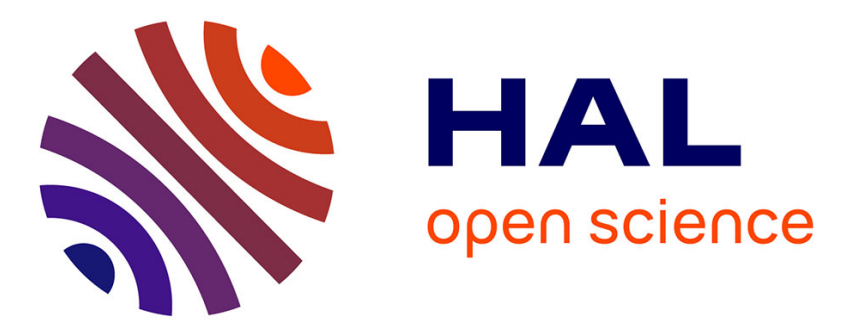

\title{
Long-range polarimetric imaging through fog
}

Julien Fade, Swapnesh Panigrahi, Anthony Carré, Ludovic Frein, Cyril

Hamel, Fabien Bretenaker, Hema Ramachandran, Mehdi Alouini

\section{To cite this version:}

Julien Fade, Swapnesh Panigrahi, Anthony Carré, Ludovic Frein, Cyril Hamel, et al.. Long-range polarimetric imaging through fog. Applied optics, 2014, 53 (18), pp.3854-3865. 10.1364/AO.53.003854 . hal-01025267

\section{HAL Id: hal-01025267 https://hal.science/hal-01025267}

Submitted on 16 Mar 2015

HAL is a multi-disciplinary open access archive for the deposit and dissemination of scientific research documents, whether they are published or not. The documents may come from teaching and research institutions in France or abroad, or from public or private research centers.
L'archive ouverte pluridisciplinaire HAL, est destinée au dépôt et à la diffusion de documents scientifiques de niveau recherche, publiés ou non, émanant des établissements d'enseignement et de recherche français ou étrangers, des laboratoires publics ou privés. 


\title{
Long range polarimetric imaging through fog
}

\author{
Julien Fade, ${ }^{1, *}$ Swapnesh Panigrahi, ${ }^{1}$ Anthony Carré, ${ }^{1}$ Ludovic Frein, ${ }^{1}$ Cyril \\ Hamel, ${ }^{1}$ Fabien Bretenaker, ${ }^{2}$ Hema Ramachandran, ${ }^{3}$ and Mehdi Alouini ${ }^{1}$ \\ ${ }^{1}$ Institut de Physique de Rennes, CNRS, Université de Rennes 1, \\ Campus de Beaulieu, 35042 Rennes, France \\ ${ }^{2}$ Laboratoire Aimé Cotton, CNRS, Université Paris-Sud 11, 91405 Orsay, France \\ ${ }^{3}$ Raman Research Institute, CV Raman Avenue, Sadashivanagar, 560 080, Bangalore, India
}

compiled: March 16, 2015

\begin{abstract}
We report an experimental implementation of long-range polarimetric imaging through fog over kilometric distance in real field atmospheric conditions. An incoherent polarized light source settled on a telecommunication tower is imaged at a $1.3 \mathrm{~km}$ distance with a snapshot polarimetric camera including a birefringent Wollaston prism, allowing simultaneous acquisition of two images along orthogonal polarization directions. From a large number of acquisitions datasets and under various environmental conditions (clear sky/fog/haze, day/night), we compare the efficiency of using polarized light for source contrast increase with different signal representations (intensity, polarimetric difference, polarimetric contrast,...). With the limited-dynamics detector used, a maximum fourfold increase in contrast was demonstrated under bright background illumination using polarimetric difference image.
\end{abstract}

OCIS codes: (110.0113) Imaging through turbid media; (110.5405) Polarimetric imaging; (010.7295) Visibility and imaging; (110.4280 Noise in imaging systems).

http://dx.doi.org/10.1364/XX.99.099999

\section{Introduction}

Imaging of objects and light sources hidden behind a turbid medium has wide applications in areas pertaining to medical diagnostics $[1,2]$, remote sensing [3] and transport and navigation [4]. More specifically, imaging through nebulous media encountered in nature, like fog, rain and light haze is still a topical issue that attracts a lot of attention. Enhanced vision in such weather conditions has indeed tremendous applications for assistance in navigation of all modes of vehicular transport. Vision systems that can offer enhanced visibility during such weather conditions can be used to provide visual assistance by means of augmented reality displays that can efficiently detect and isolate light sources hidden beyond foggy intervening media. Besides, with the advent of autonomous vehicles, both airborne and terrestrial, this problem becomes increasingly important in the field of machine vision and vehicular safety as well.

In general, the photons travelling through any

* Corresponding author: julien.fade@univ-rennes1.fr random medium can be classified into ballistic, snake and diffused photons. The diffused photons undergo maximum scattering as they travel through the medium, whereas ballistic and snake photons undergo predominantly forward scattering. As a result, the diffused photons randomly take longer paths and times to reach the detector. Consequently, in the process, they are received as noise over the ballistic photons that retain the spatial and temporal information of the source (signal). Various approaches have been studied to discriminate and record only the ballistic and snake photons in order to attain a better contrast of signal over the noise. For instance, time-gated imaging, using a gated camera synchronized to a pulsed illumination source is shown to be an efficient way to recover the information carried by ballistic photons $[1,5]$. Other approaches have also been studied such as spatial filtering techniques [6, 7], intensity modulation schemes $[8,9]$ or laser optical feedback imaging $[10,11]$.

An alternative approach, such as polarizationgated imaging [12-16], involves this time light polarization. It is based on the preservation of the 
state of polarization of light (linear or circular) during propagation through thick scattering media, such as heavy fog conditions, with minor depolarization. This so-called polarization memory effect has been analyzed in numerous references, both for linearly polarized [17] and circularly polarized light $[18,19]$. As a result, such effect can be exploited using a polarization-sensitive imaging device to enhance the visibility of a source provided this latter emits polarized light. These approaches have already proved efficient and been reported in a number of in laboratory experiments [12-16], with simulated turbidity conditions on very short distances using artificial scatterers like aerosols.

In case of light travelling through fog, photons undergo scattering by a cloud of randomly distributed particles with sizes in the order of 5-50 $\mu \mathrm{m}$ and varying number density [20]. Moreover, depending on the environmental conditions, the scatterers in the intervening medium may not retain a perfect spheroid shape due to drag (as in case of large rain drops) or may have ice crystals with preferred orientations [21].

Although these overall studies on polarized light propagation bring very useful elements of understanding, they remain quite difficult to exploit for sizing an outdoor imaging system because they are carried out in well-established and well-controlled conditions. Indeed, the combined effects of sun illumination, surrounding artificial illumination, visibility evolution, change of scatterers density and size, varying atmospheric conditions are extremely difficult to mimic or anticipate in a laboratory. In addition, unless resorting to numerical scattering simulations, long range propagation is a phenomenon that cannot be easily simulated by a laboratory experiment, even with a scaling approach.

Keeping in mind the aforementioned applications and requirement of study of such vision systems in real atmospheric conditions, we report in this paper the design, implementation and operation of an imaging experiment we developped to investigate the polarimetric contrasts of a scene including a polarized light source in real foggy environment over a kilometric distance. Such distance corresponds to a reasonable range requirement for transport safety applications. We also analyze the efficiency of several representations of the polarimetric information obtained with our imaging installation in various environmental conditions (clear sky/fog/haze, day/night).

This article is organized as follows: in the next section, we recall some basics of polarimetric imag- ing and the corresponding experimental techniques. Then, in Section 3, we detail the long-range polarimetric imaging setup used in this experiment, as well as calibration procedures in Section 4. Two representative datasets are then extensively analyzed in Section 5, among numerous datasets acquired during experimental campaigns. The efficiency of different polarimetric signal representations to increase visual contrast of a polarized light source in fog over long distance is discussed for varying environmental conditions. Then, a general discussion and conclusion on the experiment is eventually provided in Section 6 .

\section{Polarimetric contrast imaging}

Polarization sensitive imaging has proved efficient in the context of enhanced vision through turbid media [22], industrial quality control [23, 24] and machine vision [25]. To probe the complete polarimetric properties of a light source, one needs to measure the Stokes vector $S$ given by

$$
S=\left(\begin{array}{c}
S_{0} \\
S_{1} \\
S_{2} \\
S_{3}
\end{array}\right)=\left(\begin{array}{c}
I_{x}+I_{y} \\
I_{x}-I_{y} \\
I_{+45^{\circ}}-I_{-45^{\circ}} \\
I_{R}-I_{L}
\end{array}\right)
$$

from which the degree of polarization (DOP) of the source can be obtained using the relation, $D O P=\sqrt{S_{1}^{2}+S_{2}^{2}+S_{3}^{2}} / S_{0}$. Hitherto, various techniques have been employed to fully or partially measure the Stokes vectors of an image and obtain the polarimetric information of the scene of interest. Most often, rotating polarizers and/or moving birefringent plates are used. Other schemes including prisms [26], Savart plates [27], polarization gratings [28], liquid crystal modulators [29] or microgrid division-of-focal-plane polarimetric imagers [30] have been used with varying degrees of compromise towards mechanical reliability and realtime acquisition and processing. Measuring the full Stokes vector at each pixel can be a slow and storage heavy task and hence not very suitable for imaging moving objects, thus, limiting their application in real-time scenarios. In the experiment presented here, we consider a highly polarized source with a priori known linear polarization state with the intervening medium being non-birefringent. Therefore, it is not necessary to measure the full Stokes vector but only the first two components of the Stokes vector to define the so-called Orthogonal States Contrast given by Eq. (2)

$$
O S C=\frac{S_{1}}{S_{0}}=\frac{I_{\|}-I_{\perp}}{I_{\|}+I_{\perp}},
$$




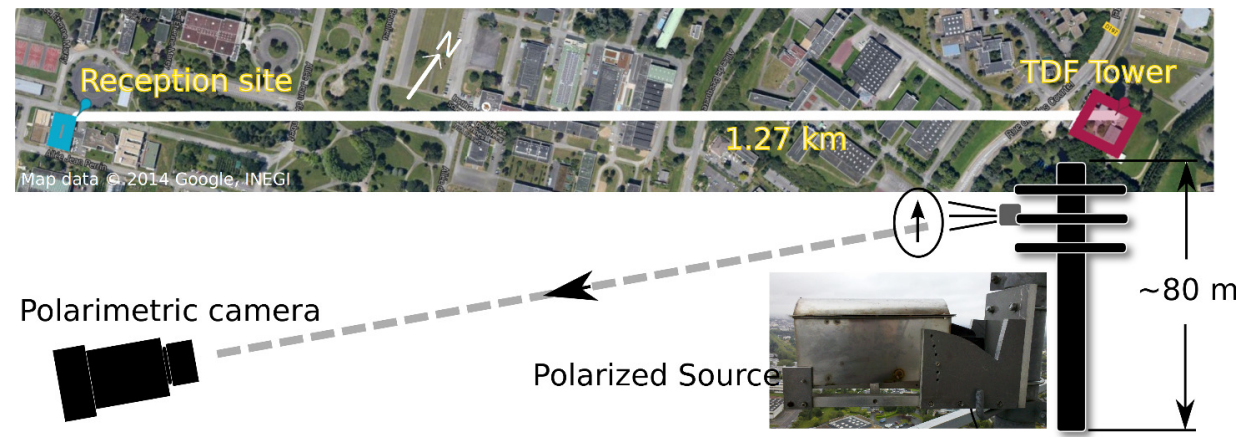

Fig. 1. Long range polarimetric imaging experimental setup. The source and the camera are separated by $1.27 \mathrm{~km}$ with the camera placed in the University of Rennes 1 campus and the source located on a telecommunication tower of the TDF company. The photograph shows the polarized light source settled on the telecommunication tower.

where $I_{\|}$and $I_{\perp}$ are the intensities obtained through orthogonally aligned analyzers or through a polarization splitting Wollaston prism. In the case considered here of a linearly polarized light propagating through non-birefringent medium, the OSC is equal to the DOP of the source after passing through the atmosphere and fog. The intensity measures recorded by a pixel of a camera are of course affected by various noise sources, for instance, (a) the Gaussian electronic noise induced by the electronic read-out circuit of the CCD array sensor ; and (b) optical noise due to photon noise, atmospheric turbulence and fluctuations introduced by spatial/temporal evolutions of the intermediate foggy medium. As will be seen later, the computation of the OSC may be strongly affected by this noise, especially in low illumination conditions, since the division by total intensity $\left(I_{T}=I_{\|}+I_{\perp}\right)$ may lead to unbounded values.

\section{Long range Experimental Setup}

The experiment presented here is designed to implement a long-range polarimetric imaging system over kilometric distance, and in real-field outdoor conditions. Such experiment allows us to assess the benefits of using polarized light for improved detectability of a light-mark through turbid atmosphere over long distances. Such a system can have practical applications in aviation or navigation at sea in poor visibility conditions. The setup mainly consists of a polarized light emitter and a polarimetric imaging system that simultaneously acquires two images of a scene corresponding to the two orthogonal directions of polarization. These elements, which are extensively described in this section, are located on the Beaulieu campus of University of Rennes 1, and are separated by $1.27 \mathrm{~km}$. The source is located on the top of a telecommunication tower owned by the
TDF company. The detection setup is located in a laboratory building as illustrated in Fig. 1. The various parts of the setup are described in detail in the following subsections.

\section{A. Polarized Source}

The source used is a $300 \mathrm{~W}$ halogen incandescent lamp with a linear polarizer, both placed inside a weatherproof steel housing as shown in Fig. 1. The light from the incandescent lamp is polarized using an adhesive dichroic polymer polarizing sheet glued to a glass plate and oriented such that the polarization axis is vertical. Half the optical power provided by the lamp being absorbed by the polarizer, it turned out that standard polaroid sheets are subject to deterioration after a few minutes of illumination. For this reason, we use polarizing sheets specifically designed for LCD projector industry, thus ensuring high durability under high power operation conditions. Before installing the source, we conducted durability tests in indoor conditions with a $300 \mathrm{~W}$ lamp. The lamp was allowed to run continuously during day times. In these conditions, it was noticed that the polarimetric contrast of the sheet was reduced by $57.4 \%$ in a period of 5 days with continuous daytime usage. In light of this, a weather-proof steel housing was designed, such that the polarizing sheet glued on a glass plate is placed sufficiently far $(20 \mathrm{~cm})$ from the lamp. Moreover, holes have been drilled in the bottom and top of the housing to enable air-flow in-between the lamp and the glass plate. This air flow is stimulated by convection when the temperature of the glass-plate increases allowing the hot air in-between to be removed, and thus providing an efficient heat dissipation. Such mechanical design ensures a reasonable lifetime of almost one year in outdoor conditions 
before requiring replacement.

The telecommunication tower is about $80 \mathrm{~m}$ in height and provides a suitable location for the source. The distance of the tower from the laboratory site in the University of Rennes 1 is optimal for various applications where a long-range imaging through fog may be crucial. The tower is also the tallest structure in the surrounding area and is in line-of-sight from laboratory buildings. The polarized source is connected to a GSM switch enabling easy remote control of the emission part of the experiment.

\section{B. Snapshot polarimetric camera}

The detection system consists of a snapshot polarimetric imaging setup and a computer dedicated for control and image acquisition. The detection system is housed on a mezzanine floor of a laboratory building in the line-of-sight of the emitter. The schematic of the imaging setup is shown in Fig. 2.(a) and 2.(b). The imaging setup consists of a telescopic arrangement of lenses L1 $(50 \mathrm{~mm}, \mathrm{~F} / 2.8$ camera objective) and L2 (25 mm, F/2.1 camera objective) which creates a collimated beam of light that is incident on a Wollaston prism (WP). The $\mathrm{WP}$ is a calcite birefringent prism with a $5^{\circ}$ splitting angle which introduces an angular separation between the vertical and horizontal polarization components of the incident beam. These ordinary and extraordinary beams are then focused onto the camera using a third lens L3 $(25 \mathrm{~mm}, \mathrm{~F} / 2.1$ camera objective), thus creating two images $\mathrm{I}_{\|}$and $\mathrm{I}_{\perp}$ on the CCD. This setup allows us to simultaneously record a scene along orthogonal polarization directions using a single camera. This has huge advantage in real-time processing of moving objects and has proved to be efficient in the presence of turbulence and relative motion of the scene [26], such as fog in our case. It has been demonstrated that this configuration suffers from lower geometrical aberration as well [26]. In order to avoid chromatic aberrations due to the WP, we use a selective red filter $(F)$ with a central wavelength of $612 \mathrm{~nm}$ and a linewidth of $12 \mathrm{~nm}$. The two images produced on the CCD have considerable overlap because of the small splitting angle of the WP. To prevent this overlap, a stainless-steel slit painted with dark matt paint of dimension $3 \mathrm{~mm} \times 18 \mathrm{~mm}$ is used as a field mask (FM) and is placed in the intermediate image plane existing between lenses L1 and L2. The CCD camera is a 12 bits, $782 \times 582$ pixels resolution camera (Basler A312f) with pixel size of $8 \mu \mathrm{m}$ and average dark count of $23 \mathrm{e}^{-} / \mathrm{s}$ with standard devi- ation of $0.6 e^{-} / \mathrm{s}$. The camera was selected for its low noise properties in low light conditions, which as we will see, is preferable while performing arithmetic on the acquired polarimetric images.
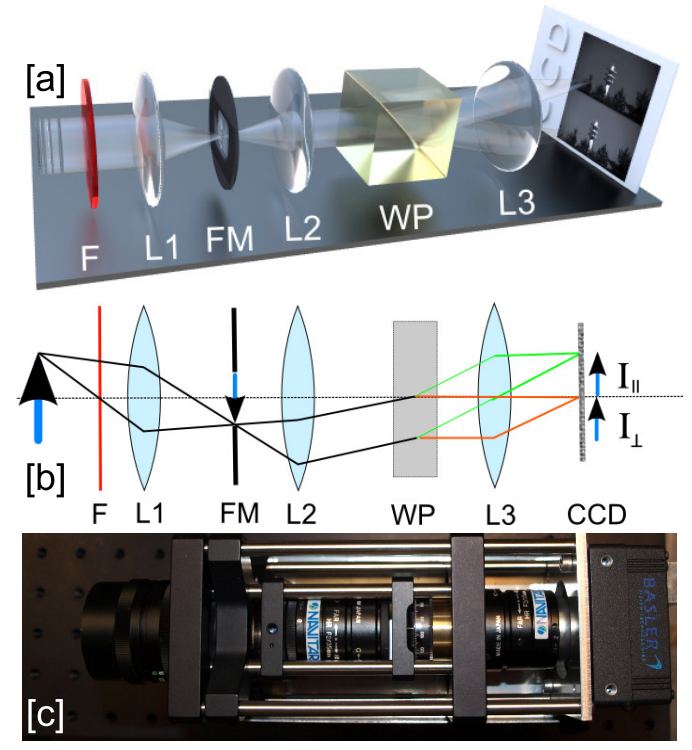

Fig. 2. Polarimetric imaging setup: The Wollaston prism (WP) angularly separates the incident beam into two orthogonal polarization components forming two images $\mathrm{I}_{\|}$and $\mathrm{I}_{\perp}$ on the CCD. The illustration in (a) shows a orthographic view of the polarimetric imager while the schematic in (b) geometrically indicates the working principle of the imaging setup. A top view photograph of the imaging setup is shown in (c).

\section{C. Control program}

The camera is controlled through an IEEE 1394a interface by a custom acquisition program developed using LabVIEW. A number of features are implemented in the program that suit the experiment and provide automation of acquisition using a user-friendly interface. One of the features implemented is what we will refer to as auto-exposure mode (AEM). We had to implement such AEM because the imaging system is by essence operated for long time periods under varying weather conditions. Under this mode, the exposure time of the camera is automatically changed depending on the illumination of a given pre-defined pixel. This pixel can be chosen to be the pixel representing the source on the camera (we will refer to this pixel as "source pixel" for brevity in further sections), thereby avoiding saturation or underexposure of the source pixel with respect to the surrounding scene. It is also possible to feed this control loop with the average brightness of a region of interest, or of the 
overall scene. The advantage of this mode is most apparent during twilight and in foggy conditions when the illumination of the scene varies strongly in time. Using dynamically controlled exposure time, one obtains a time series of frames, which can be normalized to their respective exposure times (which are stored in a data-file by the program) so as to continually exploit the full dynamic range of the camera. We also implement a so-called cumulative grab mode (CGM) to avoid recording the 100 $\mathrm{Hz}$ intensity fluctuations due to the $50 \mathrm{~Hz}$ modulation of the electrical supply network. This mode is automatically activated for exposures smaller than $10 \mathrm{~ms}$, that is when the source blinking becomes apparent. Under CGM mode, a frame having maximum gray-level value of the source pixel is chosen from a sample of $N_{\mathrm{CGM}}$ frames where $N_{\mathrm{CGM}}$ depends on the exposure time $T_{\exp }$ in milliseconds as $N_{\text {CGM }}=20(\mathrm{~ms}) / T_{\text {exp }}$. The program also displays the calculated OSC of the source pixel in real-time and allows for compensation of the ambient light contribution, in which case the OSC reads

$$
O S C_{A}=\frac{\left(I_{\|}^{s}-I_{\|}^{a}\right)-\left(I_{\perp}^{s}-I_{\perp}^{a}\right)}{\left(I_{\|}^{s}-I_{\|}^{a}\right)+\left(I_{\perp}^{s}-I_{\perp}^{a}\right)} .
$$

In the above equation, $I_{\|}^{s}$ (respectively $I_{\perp}^{s}$ ) denotes the average intensity over a $3 \times 3$ pixels area enclosing the pixel corresponding to the source location (source pixel) in $I_{\|}$(respectively $I_{\perp}$ ). On the other hand, $I_{\|}^{a}$ (respectively $I_{\perp}^{a}$ ) estimates the ambient illumination by averaging a $3 \times 3$ pixels area in the vicinity of the source pixel, but strictly distinct from the source spatial extent. With such definition, the OSC is computed taking into account intensity measures $I_{\|}^{a}$ and $I_{\perp}^{a}$ of a reference area close to the source representing the ambient illumination.

Using such control software with AEM allows us to acquire images at predefined intervals over long periods of time with strongly varying illumination conditions (i.e., clear sky, obstructed vision, daytime/night-time and twilight).

\section{Experiment calibration}

In this section, we first describe the camera calibration and image registration procedures which have to be implemented so as to provide reliable polarimetric images and measurements. Then, we report some experimental results which allowed us to validate the experimental system (emission and acquisition) and laboratory calibration on real field conditions.

\section{A. Imaging setup calibration}

The frames obtained in the experiment described above are binary values obtained from the camera. Processing of the frames to extract the images is at this step carried out in a post-acquisition stage. The post-processing includes fixed pattern dark frame subtraction, pixel non-uniformity correction, image registration and optical distortion correction. The latter processing steps are organized in the following manner.

Detector calibration: For a camera sensor there are mainly three calibrations to be performed in order to record an image that closely corresponds to the scene being imaged, namely dark pattern calibration, non-uniformity of pixel gain and removal of hot and dead pixels. Each pixel has different dark noise properties at a particular temperature and exposure time. After averaging over a number of dark frames acquired in total darkness, one obtains the fixed pattern dark frame $\left(\mathrm{I}_{F P D}\right)$ that can be subtracted from the images of interest. It must be noted that this correction depends on the exposure time. We thus averaged 500 dark frames to obtain the $\mathrm{I}_{F P D}$ for different exposure times. Another important correction to be made is the nonuniformity in pixel gain. Owing to the fabrication process, each pixel in the sensor may not have the same sensitivity/gain. This non-uniformity is estimated by illuminating the sensor with spatially uniform white light and recording the resulting pattern. This image is normalized by dividing by the mean gray level to create a gain non-uniformity image $\mathrm{I}_{G N U}$. Using the above two calibration images, a raw image recorded is systematically corrected using $\mathrm{I}_{\text {corr }}=\left(\mathrm{I}_{\text {raw }}-\mathrm{I}_{F P D}\right) / \mathrm{I}_{G N U}$.

Image registration and distortion calibration: Once performed the above calibrations and corrections on the bare detector itself (without any image forming optics), the polarimetric optical imaging system is mounted. When the system is assembled and images are recorded, one has to extract the two images corresponding to orthogonal polarization directions from a single frame. It is required that the two extracted images have a one to one correspondence between pixels such that they map the same scene. This process is straightforward if there is no geometrical distortion or if both the images share the same geometrical distortion. However, the WP used in the polarimetric imaging setup does introduce astigmatism and anamorphic distortion in the resulting images [31]. The distortion is non-symmetrical with respect to the two image channels and hence a perfect image registration 


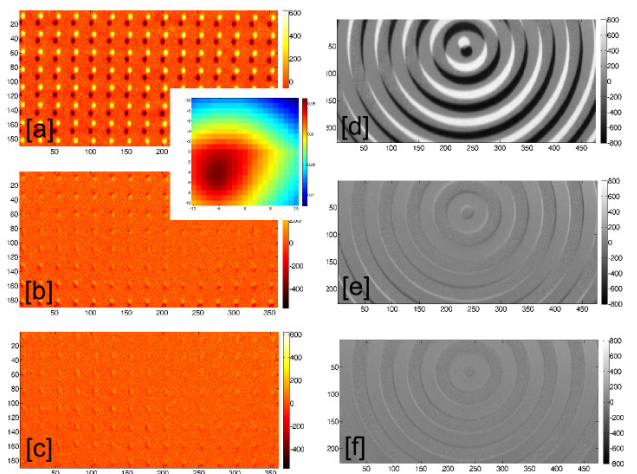

Fig. 3. Difference image $\left(\mathrm{I}_{\|}-\mathrm{I}_{\perp}\right)$ of the calibration target (grid of dots) (left) and test object (right). The top images correspond to the difference images formed with approximate extraction of the two image channels. The middle row is the result of image registration correction by pixel linear translation, and the bottom row corresponds to the difference images created after applying the distortion correction algorithm. The inset shows the correlation map obtained by translating the $\mathrm{I}_{\perp}$ over $21 \times 21$ pixels and finding maximum correlation with $\mathrm{I}_{\|}$.

is not possible by merely translating one of the image to match the other. The difference in distortion in the two images can be reduced by a non-linear coordinates transformation on $\mathrm{I}_{\perp}$ to match the distortion in $\mathrm{I}_{\|}$.

To perform such corrections, a grid of equally spaced dots (printed black dots on white paper with no specific polarization orientation) was imaged through the system and the acquired images were used to calibrate the two image registration methods, namely simple translation or non-linear coordinates transformation. The correction by translation was obtained by a maximum correlation technique where one of the image channels, say $\mathrm{I}_{\perp}$, was translated in $\mathrm{X}$ and $\mathrm{Y}$ directions over $21 \times 21$ pixels and correlation values with $\mathrm{I}_{\|}$were recorded for each translation position. Then, a best match was found by looking for the maximum correlation value and translating $I_{\perp}$ accordingly. The inset in Fig. 3 shows the correlation map and Fig. 3.(b) shows the difference image $\mathrm{I}_{\Delta}=\mathrm{I}_{\|}-\mathrm{I}_{\perp}$ after correction by translation. This shows a great improvement in comparison to uncorrected images (Fig. 3.(a)), but it can be observed that pixel registration is imperfect in the outer regions of the image.

Therefore, to digitally remove the residual mismatch caused by the non-symmetrical aberrations in both channels, we resort to a non-linear coordinates transformation on the pixels of image $I_{\perp}$ to match the distortion in $\mathrm{I}_{\|}$. This is performed by extracting the coordinates of the centroids of the dots in two thresholded polarimetric images and finding the coefficients of a $2 \mathrm{D}$ polynomial of degree 3 for the X-Y coordinates transformation. The coefficients of the polynomial are found using least squares optimization procedure. A polynomial of degree three has been found to be sufficient to describe the non-linear transformation as the higher order coefficients of the polynomial are negligible compared to the lower order coefficients. By applying this non-linear coordinates transformation to the pixels of $I_{\perp}$, we obtain a distorted version of the latter, which now shares the same geometrical distortions as $I_{\|}$. For further processing of the polarimetric images, it is lastly necessary to resample the resulting image on the same regular (square) set of pixels locations as $\mathrm{I}_{\|}$, which is done by a Matlab implementation of a standard bicubic interpolation algorithm. The result of this geometrical correction procedure can be observed in the difference image after correction given in Fig. 3.(c).

A comparison of effectiveness of the calibration and of the image registration using simple translation and non-linear correction is given in Fig. 3.(df) on a test object (plain white paper with circular pattern in black). In comparison to approximate extraction (Fig. 3.(d)), simple translation allows for a reduction by $63 \%$ of the span of extreme values in the difference image (Fig. 3.(e)), whereas with the non-linear distortion correction, a further reduction by $30 \%$ is obtained (Fig. 3.(f)).

It must be noted that the non-linear correction method is computationally slower than finding linear translation parameters by maximum correlation method. The calculation of parameters for nonlinear correction is a one-time calibration procedure that takes about a minute for completion on a standard computer (Matlab on $2.60 \mathrm{GHz}$ processor). This time is acceptable even though it is 25 times slower than finding maximum correlation in a small neighborhood of $21 \times 21$ pixels. However, the computational time required to correct each frame becomes very stringent for real-time applications. In this case, the simple translation is four orders of magnitude faster than the non-linear correction, which takes about 2 seconds to process because of interpolation of a large number of pixels values. As a consequence, for applications requiring high processing speed and where only a small region of the image is of interest, it is sufficient to use only linear translation. Thus, in the remainder of this article, as we shall consider a small region of interest 
around the source location, we will limit ourselves to simple translation registrations, for the sake of computational effectiveness on long time series experiments.

Intensity Asymmetry: It is important to take note of any transmission asymmetry in the two image channels introduced by the WP. In our case a $2.7 \%$ asymmetry in intensity was observed after imaging a uniformly illuminated depolarizing object (plain white paper observed with different orientations) through the system. The measured average intensity ratio $\left(\mathrm{I}_{\|} / \mathrm{I}_{\perp}=1.027\right)$ was used to correct this asymmetry. This ratio was seen to be constant over a large central region including the region of interest.

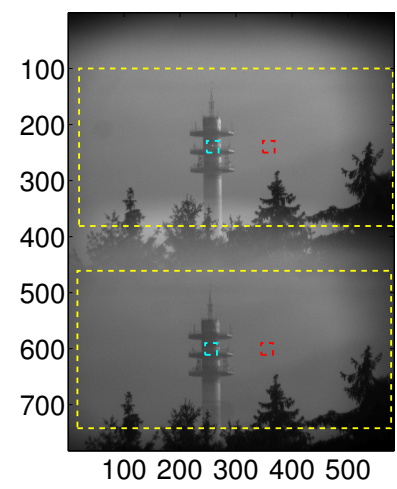

Fig. 4. The raw image obtained from the camera with $782 \times 582$ pixels. Extraction of two image channels after applying image registration process is demarcated in yellow dashed box. The ambient illumination is obtained by averaging over the region shown in red-dashed lines and the region shown in blue dashed lines is the $21 \times 21$ pixels ROI that includes the source at its center.

\section{B. In-situ verification}

Along with the above calibration procedures, further alignment of the detection system with the polarization direction of the source is done while mounting the detection system. The polarimetric camera setup and the control/acquisition computer are settled in a mezzanine floor of a building facing the telecommunication tower. It has first been checked that the spread of the light source was close to our expectancies $(1 \times 1$ pixel with the selected $50 \mathrm{~mm}$ focal length objective lens), with an actual horizontal spread of 1.5 pixels and vertical spread of about 2 pixels. The main discrepancy in the vertical direction is due to the influence of the WP.

The whole setup is placed on a stable mechanical mount with adjustable angle (pitch) so that the imaging system can be aligned in line-of-sight with the source on the tower. In addition, the tilt angle (roll) must be well aligned so that the polarization axis of the imager is strictly aligned with the polarization direction of the source. This calibration is preferably done during night time in clear sky with the polarized lightmark turned on. It is indeed easier to minimize and even null the intensity at the source location in the perpendicular polarization image $\left(\mathrm{I}_{\perp}\right)$ in dark background. After such calibration, we observed an OSC of 0.97 for the source pixel which increased to a value of 1.00 when OSC was computed taking into account the low intensity background contribution, as in Eq.(3).

During these preliminary calibration acquisitions, it was checked on the acquired frames that the daytime clear sky is polarized to some extent, depending on sun location, and that the ambient light backscattered on the tower structures and imaged on the camera can partly retain polarization. However, we observed that the imaged scene is almost totally depolarized as soon as a thin layer of fog or haze is present in the atmosphere, or when the weather is covered by (even very thin) clouds. As a result, the difference image of the two image channels is expected to provide an obvious advantage over intensity imaging due to its ability to cancel the unpolarized background and isolate the polarized source.

\section{Experimental results: Signal contrast in foggy condition}

Once settled, optimized and calibrated the imaging system described in previous sections, and using the above mentioned control program, we were able to acquire numerous series of frames of the source at different times of the day (dawn, daytime, dusk, nigthtime) with varying atmospheric conditions (clear sky, thin haze, fog, cloud, rain, snow,...). The corresponding time-series acquisitions were recorded during years 2011 and 2012 with various time periods (10 images/minute to 6 images/hour) over long time spans of several hours or tens of hours.

During these long term acquisitions, the dynamically varying exposure time in AEM was recorded for each acquisition and the frames were normalized to their respective exposure times during postacquisition analysis. From each raw frame, we then extracted the two sub-images $\mathrm{I}_{\|}^{j}$ and $\mathrm{I}_{\perp}^{j}$ (with $j$ corresponding to the $j^{\text {th }}$ image in the time series). One raw image extracted from a time series is represented in Fig. 4, with an overlay indicating the image registration (rectangles in yellow) from which 
Signal representation Symbol Definition

\begin{tabular}{ccc}
\hline Intensity & $\gamma_{I}$ & $I_{\|}+I_{\perp}$ \\
Polarimetric difference & $\gamma_{\Delta}$ & $I_{\|}-I_{\perp}$ \\
OSC & $\gamma_{O S C}$ & $\frac{I_{\|}-I_{\perp}}{I_{\|}+I_{\perp}}$ \\
Polarimetric ratio & $\gamma_{\text {ratio }}$ & $\frac{I_{\|}}{\left\langle I_{\perp}\right\rangle_{\chi_{k}}}$
\end{tabular}

Table 1. Signal representations of polarimetric data, with \langle\rangle$_{\chi_{k}}$ denoting spatial averaging over a square neighbourhood $\chi_{k}$ of the central pixel of size $N_{k}$ pixels.

regions of interest (ROI) corresponding to $\mathrm{I}_{\|}^{j}$ and $\mathrm{I}_{\perp}^{j}$ are extracted. The example of ROI shown in light blue in Fig. 4 is a $21 \times 21$ pixels rectangle surrounding the source location.

\section{A. Principle of the measurement}

These numerous acquisitions were used to gauge the effectiveness of polarimetric imaging under various atmospheric conditions. The analysis of the experimental results led us to retain four main different scenarios, associating clear sky / foggy conditions and day/night time. The conditions in daytime with fog are indeed such that the intensity of the pixels around the source are nearly uniform, making it difficult to identify the source from the background in a simple intensity image. In clear sky conditions, the background consists of the telecommunication tower structures and installations. On the other hand, in night time we have conditions where the source is brighter and the background is dark with unpolarized and localized bright light sources (for instance, the red aircraft warning lights on the tower) or sometimes, during heavy fog, a uniformly lit background because of scattering of city lights.

In all these conditions, we are interested in maximizing the visual contrast of the source with respect to its background. Polarimetric imaging provides us with two data sets $\left(I_{\|}\right.$and $\left.I_{\perp}\right)$ for the same image and thus allows us to create various representations of the image with different visual contrasts. We thus propose to consider four simple signal representations of the available data, generally referred to as $\gamma$, and compare their visual contrast under various environmental conditions. These four signal representations correspond to a simple intensity image $\left(\gamma_{I}\right)$, a polarimetric difference image $\left(\gamma_{\Delta}\right)$, the OSC image $\left(\gamma_{O S C}\right)$ and a polarimetric ratio image $\left(\gamma_{\text {ratio }}\right)$, and are given in Table 1 , along with their respective symbol and mathematical definition.

It is obviously seen that the absolute values of these signal representations are different. However, we will be interested in the remainder of this section in comparing the visual contrast of the polarized source that can be obtained with these different representations. We must therefore provide a fair comparison between the contrasts obtained in different representations. For this purpose we define a contrast-to-noise ratio (CNR) such that the CNR value is insensitive to the scale of these four representations. The CNR is defined for a central pixel denoted $s$ (signal $\gamma_{s}$ ) with respect to its surrounding square neighbourhood $\chi_{k}$ of size $N_{k}$ pixels as,

$$
C N R=\frac{\gamma_{s}-\langle\gamma\rangle_{\chi_{k}}}{\sqrt{\frac{1}{N_{k}-1} \sum_{i \in \chi_{k}}^{N_{k}}\left(\gamma_{i}-\langle\gamma\rangle_{\chi_{k}}\right)^{2}}}
$$

where $\langle\gamma\rangle_{\chi_{k}}=\sum_{i=1}^{N_{k}} \gamma_{i} / N_{k}$ is the spatial average over $\chi_{k}$. It must be noted that the evaluation of the spatial averages are carried out for a sample of pixels defined by the region $\chi_{k}$ that excludes the $2 \times 2$ central region centered on the source pixel to prevent spatial extent of the light source from perturbing computation of the background statistical properties. With the above definition, the CNR is basically the measure of how many standard deviations away does the signal pixel lie from the distribution of its surrounding pixels. The CNR values computed on the data provide a more quantitative evaluation of the contrast than a visual analysis of the polarimetric images plotted in subsequent figures. Moreover, in an applicative context, the CNR would be directly linked to the performance of automated detection of polarized sources of unknown number and locations in a scene. In the presence of structured background and/or unpolarized spurious sources, a high CNR would warrant good detection probability along with low false alarm rate.

In the following subsections, we provide a thorough data analysis performed on two particular time-series acquisitions, allowing one to observe clear sky and foggy conditions in day time (Subsection 5.B) and night time (Subsection 5.C). These two experimental data series are representative of the wide variability of environmental conditions that were made possible to address with the experimental facility described in this article.

\section{B. Day time measurement}

This first data acquisition series that we will focus on has been recorded on the 17-Oct.-2011, between $10.02 \mathrm{am}$ and $10.57 \mathrm{am}$. During this 55-minutes acquisition, an image has been recorded every 10 seconds, making it possible to observe rapid rising 
[a]

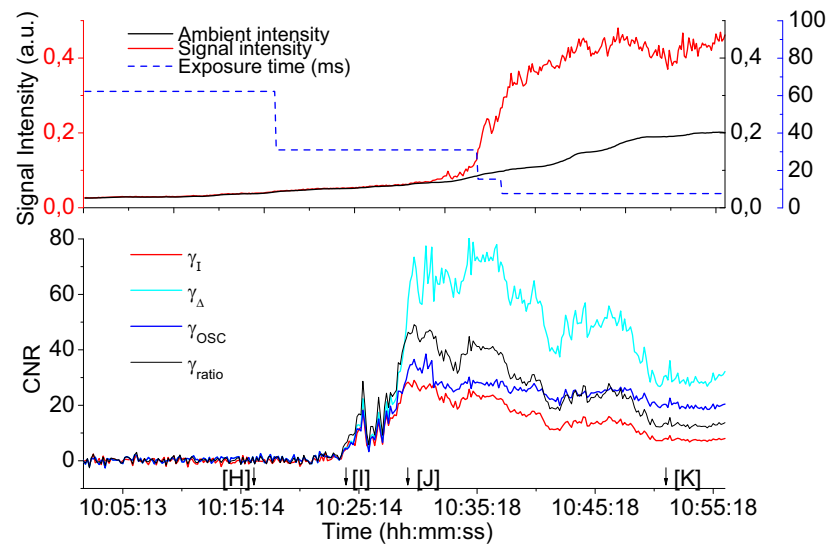

[c]

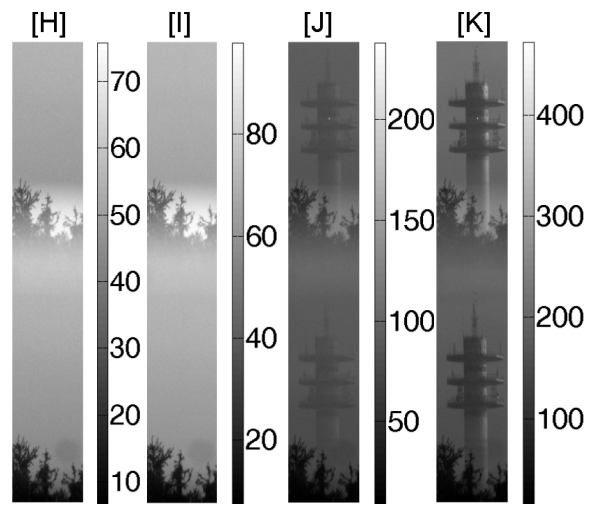

Fig. 5. (a) Time evolution of the source (signal) intensity, ambient intensity and camera exposure time across day time experiment. (b) Time-evolution of the CNR of four polarimetric signal representations. (c) Raw images of four frames labelled H, I, J and K, corresponding to various visibility conditions across day time experiment.

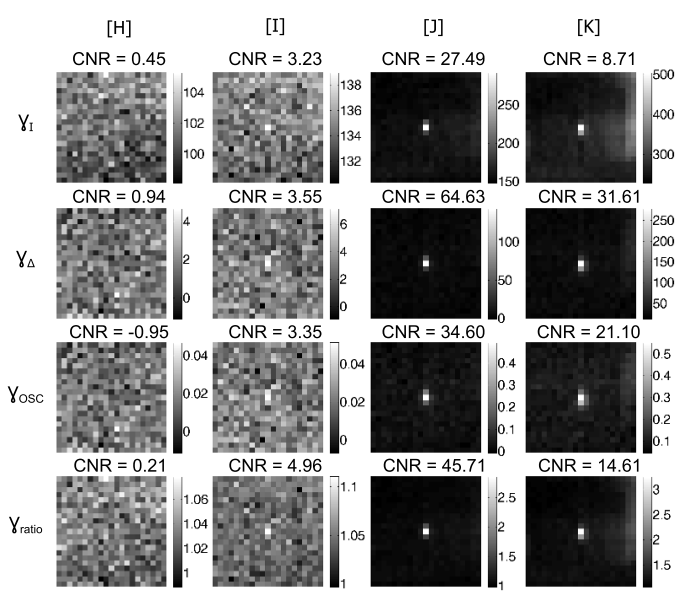

Fig. 6. CNR images of the $21 \times 21$ region of interest around the polarized light source, extracted at times indicated by H, I, J and K in Fig. 5. Row 1 to 4 correspond to the CNR obtained with intensity image, difference image, OSC image and contrast ratio image respectively. The corresponding CNR for the central source pixel are also given as numerical figures for comparison.

of a thick fog and evolution of its density within a period of 30 minutes. The evolution of the experimental conditions can be observed in Fig. 5. In Fig. 5.(a), the intensity of the pixel corresponding to the location of the polarized source (signal pixel) is plotted in red curve as a function of time. In addition, the evolution of the ambient intensity is represented in black curve. This quantity was estimated from a spatial averaging of the total intensity $I=I_{/ /}+I_{\perp}$ in a homogeneous $21 \times 21$ pixels sky region, marked in Fig. 4 with a red square. The cam- era exposure time during the whole experiment was controlled by the software in Auto Exposure Mode, so as to prevent underexposure or saturation of the signal pixel. The evolution of the exposure time is also provided in Fig. 5.(a) in dotted blue curve, revealing the smooth evolution of the fog density and background illumination during the experiment.

Across this experimental dataset (330 images), four instants will be considered, labeled $\mathrm{H}, \mathrm{I}, \mathrm{J}$ and $\mathrm{K}$ in Fig. 5.(b). The corresponding raw images acquired by the imager are respectively plotted in Fig. 5.(c). They correspond to different interesting situations of visibility of the source through fog. The first situation $\mathrm{H}$ corresponds to high fog density where the source is completely embedded in the background illumination, as can be checked in Fig. 5.(a) by comparing the levels of the signal and background intensities. Acquisition I is the first image recorded on which the light source begins to be detectable over the background. Then, acquisition $\mathrm{J}$ corresponds to an intermediate situation of a light haze limiting the visibility of the source whereas the last measurement $\mathrm{K}$ has been acquired in clear sky, but with a significantly higher background illumination than in the $\mathrm{J}$ situation.

Let us now analyze the CNRs associated to the four signal representations considered in this article and defined in Table 1. The evolution of the CNRs across the whole dataset is given in Fig. 5.(b). In Fig. 6, we also provide the contrast images computed for each signal representation and for each situation $\mathrm{H}-\mathrm{K}$, on a $21 \times 21$ pixels neighbourhood of the central signal pixel. The numerical values 
indicated in Fig. 6 correspond to the CNR of the central pixel estimated on this neighbourhood. As can be seen from these results, the source is hidden behind heavy fog until 10.13 am and all CNR values remain close to zero in this situation. It can be indeed checked in Fig. 6 that the source is not distinguishable on any of the four signal representations studied.

Measurements performed after 10.13 am are more interesting. Indeed, they first allowed us to observe that, as soon as the light emitted by the source is visible over the background, it retains a very high polarization degree, with no detectable depolarization induced by turbid atmosphere, nor partially depolarized halo in the vicinity of the source location. Following a similar interpretation as in prior laboratory experiments [12-17] on short distances, this result can be understood considering properties of Mie scattering on large particle size in fog droplets $[16,32]$. In our case, since we are working at a very long distance, the light emitted by the source can be almost totally absorbed by a thin fog, which in return cannot be considered as a strongly diffusive medium, and hence cannot induce strong depolarization. In addition, the low numerical aperture of the imaging system at such distance acts as a spatial filter $[6,7]$, thus preventing most of the scattered and snake photons (slightly scattered) from entering the imaging system.

Then, the results given in Figs. 5 and 6 reveal that, as soon as intensity coming from the source is detectable, all polarimetric signal representations $\left(\gamma_{\Delta}, \gamma_{O S C}\right.$ and $\left.\gamma_{\text {ratio }}\right)$ provide CNR values significantly higher than the CNR obtained with a standard non-polarimetric intensity measurement $\left(\gamma_{I}\right)$. This general result thus confirms the interest of polarimetric measurements for enhanced vision through poor visibility conditions. The analysis can be however refined so as to identify the best signal representation in a given situation. In very low visibility conditions (acquisition I), it appears that $\gamma_{\text {ratio }}$ gives the best improvement in terms of CNR (60\% increase) with respect to an intensity measurement $\gamma_{I}$.

On the other hand, when visibility increases, the best signal representation corresponds to a difference image $\gamma_{\Delta}$ allowing the CNR to be enhanced up to a factor of almost 4 (acquisition $\mathrm{K}$ ). The efficiency of the difference image is due to its ability to suppress the background contribution. This enhancement is either due to diffused light in the turbid atmosphere, as in acquisition $\mathrm{J}$, or due to the surrounding scene, as in acquisition $\mathrm{K}$ on which ele- ments of the telecommunication tower can be identified on the intensity image. Indeed, as it has been noticed in Subsection 4.B, the ambient light does not exhibit noticeable polarization as soon as the sky is covered by clouds or when a thin layer of haze or fog is obscuring the atmosphere. Hence, the contributions of this ambient background on the two polarimetric images $I_{/ /}$and $I_{\perp}$ are strictly identical, and can be suppressed on a difference image.

Lastly, it is interesting to note that $\gamma_{O S C}$ never appears to be the best representation, contrary to anterior work on active polarimetric imaging $[33,34]$ (where, due to the reflection geometry of imaging, the background has a significant polarized component). Indeed, in the present context of active polarized source and passive totally unpolarized background, OSC implies a normalization of $\gamma_{\Delta}$ by the total intensity $\gamma_{I}$, which, on the dark background image $\gamma_{\Delta}$ obtained, increases the relative noise in the final image and thus decreases the CNR. In the next subsection, it will be shown that such normalization can be much more detrimental to signal quality in night time conditions. Therefore, this normalization must be avoided when it comes to locating or detecting a target in poor visibility conditions, unless one wants to assess the DOP of the light source.

\section{C. Night time measurement}

A similar experiment was conducted during night time on the 14-Oct.-2011, between 1.53 am and 7.17 am. During this 324 minutes acquisition, a raw frame has been recorded every minute. As in the previous subsection, the evolution of signal pixel and background intensities are plotted respectively in red and black curve in Fig. 7.(a). The evolution of the exposure time over this long time period is given in Fig. 7.(a) in dotted blue curve (logarithmic scale). This curve shows the strong variations of the exposure time at night with atmospheric conditions, ranging from $7 \mathrm{~ms}$ to a maximum value of $4 \mathrm{~s}$ authorized by the camera used. From the observation of these curves, it can be noted that a dense fog cloud has totally obscured the visibility of the source between $3.40 \mathrm{am}$ and $4.55 \mathrm{am}$, leading to a maximum exposure time in total darkness, as in situation labelled $\mathrm{M}$ in Fig. 7. On the other hand, the beginning and end of the acquisition correspond to almost clear sky conditions (as in situations labelled $\mathrm{L}$ and $\mathrm{O}$ ). The strong signal intensity variations observed in clear sky conditions are mainly due to atmospheric turbulence which causes flicker of the location of the signal pixel on the images, and 
[a]
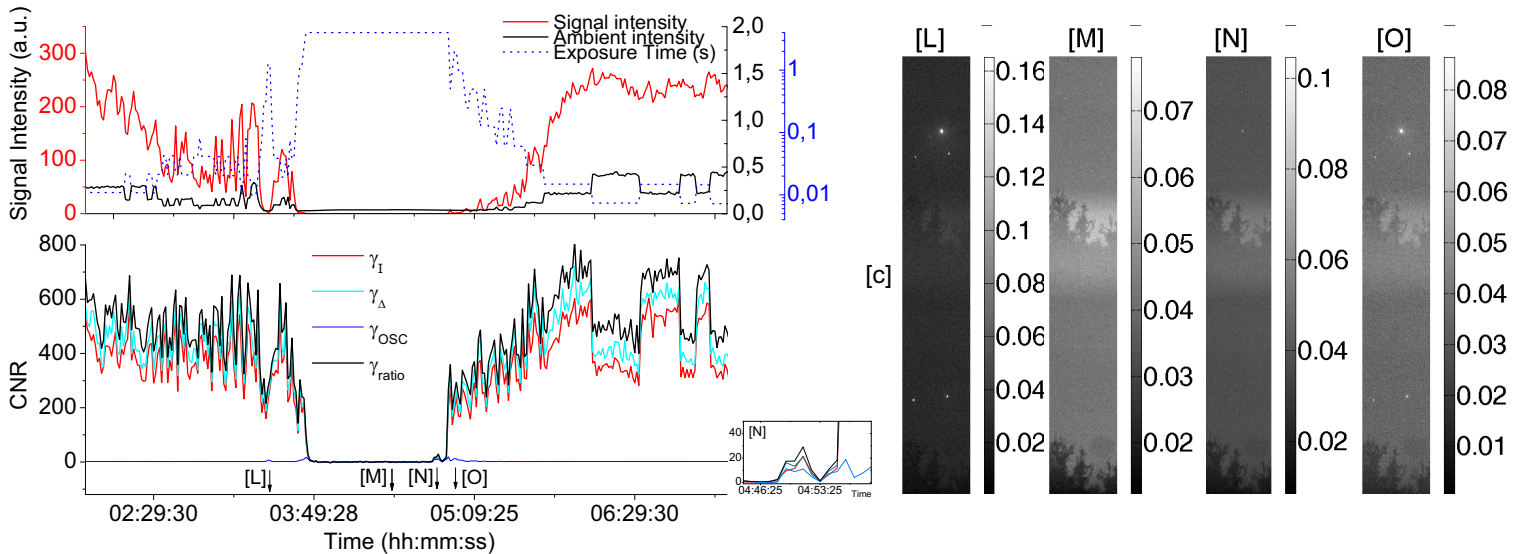

Fig. 7. Same as Fig. 5 for night time experiment. The inset shows a magnified part of the plot where the fog is thinning and the CNRs are comparable for all signal representations. Four frames labelled L, M, N and O have been selected to be representative of visibility conditions during night experiments. The display dynamics in (c) has been expanded by a factor of 20 for all night situations so that the background becomes visible.

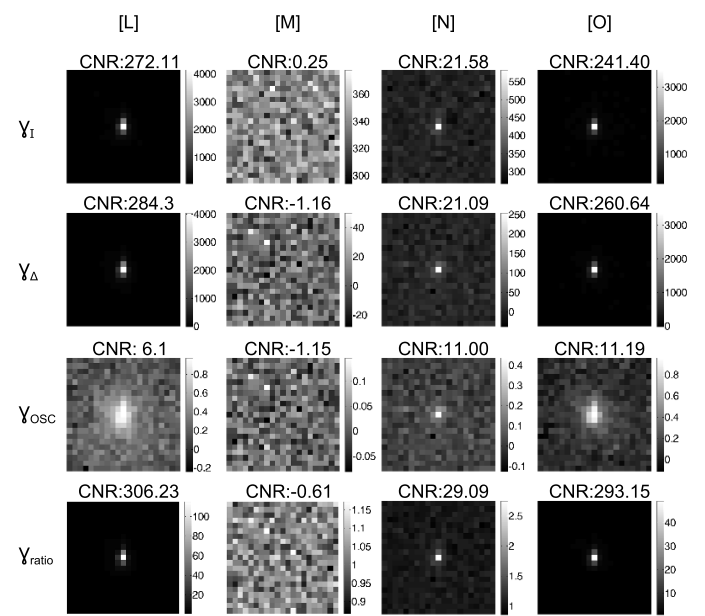

Fig. 8. Same as Fig. 6 for night time experiment. The OSC image is observed to be noisy and has least signal contrast as a result.

can also be partly due to an unperfect smoothing of the source intensity modulation at $100 \mathrm{~Hz}$. In the conditions of the experiment, one other situation labelled $\mathrm{N}$ has retained our attention since it corresponds to one of the rare instants of fog setting/rising in which the source is partly visible. The raw images corresponding to these four situations are also plotted in Fig. 7.(c).

As in Fig. 5, the CNRs associated to the four signal representations are plotted in Fig. 7.(a) across the whole dataset. The contrast images obtained at each particular situation analyzed L, M, N and $\mathrm{O}$ are given in Fig. 8. These experimental results first confirm that OSC is not a relevant signal representation due to noise amplification occurring with normalization. The corresponding CNR is indeed always close to zero, and the noise amplification can be clearly observed in Fig. 8. This effect is particularly enhanced in night conditions since background values are very close to or equal to zero (since the exposure time is limited in clear sky to prevent saturation of the source pixel, see Fig. 7.(a)), thus leading to non-numerical values in the final image, which are not taken into account in the computation of CNR.

The comparison of the three other signal representations considered in this paper is more informative. It can first be noted that between 3.40 am and $4.55 \mathrm{am}$, none of the representations allows the source to be significantly detected over the background, due to total absorption of the emitted light when propagating through dense fog. In clear sky conditions however (situations L and O) or partially reduced visibility (situation $\mathrm{N}$ ), it can be observed that simple intensity image and polarimetric difference image share comparable CNR values. This confirms the fact that $\gamma_{\Delta}$ only outperforms a simple intensity image in the presence of significant background contribution, as observed above in daytime conditions. In night time conditions, the very dark background is strongly affected by camera dark noise and a difference image could, in some configurations of noise realization, lead to a degradation of the CNR with respect to an intensity detector. Only the ratio representation $\gamma_{\text {ratio }}$ allows 
improving the CNR values by a factor comprised between $10 \%$ and $35 \%$, which can be explained by the noise reduction induced by the spatial averaging of the background in the expression of $\gamma_{\text {ratio }}$.

These results, which seem to soften the interest of polarimetric imaging for vision enhancement though turbid atmosphere in night conditions, actually reveal the limitations of the sensor used for these experiments. These nocturnal acquisitions indeed allow us to identify two ways of improvement for our detection system, which are (a) the minimization of the dark noise, and most importantly (b) the increase in detector dynamics. Reducing the dark noise would limit the level of noise in dark background in night-time conditions for polarimetric signal representations, as stated above. Moreover, it can be observed in Fig. 7.(b) that between $6.00 \mathrm{am}$ and $7.10 \mathrm{am}$, strong variations in all the CNR values occur, following the same abrupt "steps" at each modification of the exposure time. This is a confirmation that in these situations, the dark image background is perturbed with camera dark noise. Usually, the dark noise level increases with the acquisition time. However, the Basler A312f camera used implements a Sony ICX 415AL sensor, whose HAD (HoleAccumulation Diode) technology makes it possible to inhibit the increase of dark noise level with integration time. As a result, the noise contribution is thus artificially doubled in our measurements, relatively to the signal pixel value when exposure time is divided by two, hence leading to a strong decrease in terms of CNR. This also confirms that adapting the exposure time cannot increase the dynamics of a detector when the illumination variations across the scene exceed the actual sensor dynamics. It would thus be highly beneficial to use a high dynamics sensor to improve these results and potentially increase the range of environmental conditions for which polarimetric imaging could be advantageous for vision enhancement through turbid atmosphere over long distances.

\section{Conclusion}

In this paper, we have reported an experimental setup allowing to perform snapshot polarimetric contrast imaging of a light emitter through turbid atmosphere over kilometric distance. With this setup it has been possible to acquire long timeseries of measurements in various real environmental conditions of weather and visibility. The results reported in the article have permitted to assess the benefits of using a polarized light source and a polarization-sensitive camera to increase vi- sual contrast or detectability of such light source in degraded visibility conditions, and at a distance compatible with potential applications, such as navigation assistance for airborne, maritime or terrestrial transportation. It can be concluded from these results that, for the purpose of detecting a polarized source embedded in an unpolarized background, using polarized beacons and polarimetric cameras can lead to a significant improvement of the contrast. This is particularly true in the presence of high background illumination levels, which is likely to occur in daytime conditions, or in night-time situations, due to scattering of unpolarized ambient light by the atmosphere itself. This is indeed beneficial, as computing a simple polarization-difference image would make it possible to isolate the target from ambient light and hence provide higher contrast in foggy situations in real-time.

Another result of this article is that no significant depolarization of the polarized source, nor depolarized halo could be detected during propagation through fog over a kilometer with a standard dynamics camera, as the one used in this experiment. This observation, unreported to our best knowledge at a kilometric distance, thus confirms the interest of conducting such experiments in realfield conditions, over long distances. This behavior, similar to the one observed on short distance laboratory experiments was not an entirely predictable result. It is indeed difficult to mimic the physical conditions that were encountered during these experiments with in laboratory short distance measurements using highly diffusive water or gas tanks.

As a perspective to this work, we are currently implementing to our setup a new camera with a higher dynamics range and lower noise level, so as to refine the first analyses presented in this article. Such a camera will make it possible to analyze situations in which observations were limited by the detector itself. More importantly, it is also expected that a high dynamics sensor will increase the range of application of polarimetric imaging in terms of visibility distance for beacon detection or localization in turbid atmosphere. Other important perspectives to this work can be enumerated, among which the definition of clear figures of merit that link image contrast ratios with visibility range. Another important field of research that will be addressed is the definition of optimal signal representation as a function of environmental or instrumental conditions for various image processing tasks, such as estimation or detection. Recent results have evidenced the potential of polarimetric 
imaging contrast when relevant statistical parameters, such as partial correlation between measurements, are considered [35]. Applying these theoretical results to experimental data will necessitate a more thorough analysis of the images in terms of statistical properties, which will also be enabled with the use of a better camera. Then, the implementation of these optimal estimators/detectors on FPGA boards for in-line real-time image processing and display is also an interesting perspective to this work.

\section{Acknowledgments}

The authors are grateful to the TDF company, and in particular to A. Lecluse and P. Gelebart, for providing location for the emitter on the telecommunication tower. This work has been partly funded by the CEFIPRA (project $\mathrm{N}^{\circ}$ 4604-4).

\section{References}

[1] S. Andersson-Engels, R. Berg, S. Svanberg, and O. Jarlman, "Time-resolved transillumination for medical diagnostics," Optics letters 15, 1179-1181 (1990).

[2] Z. Nan, J. Xiaoyu, G. Qiang, H. Yonghong, and M. Hui, "Linear polarization difference imaging and its potential applications." Applied optics 48, 67346739 (2009).

[3] M. Dubreuil, P. Delrot, I. Leonard, A. Alfalou, C. Brosseau, and A. Dogariu, "Exploring underwater target detection by imaging polarimetry and correlation techniques," Applied optics 52, 997-1005 (2013).

[4] W. R. Watkins, D. H. Tofsted, V. G. CuQlockKnopp, J. B. Jordan, and J. O. Merritt, "Navigation through fog using stereoscopic active imaging," in "Enhanced and Synthetic Vision 2000, SPIE Conference Series," , vol. 4023 (2000), vol. 4023, pp. 20-28.

[5] L. Wang, P. Ho, C. Liu, G. Zhang, and R. Alfano, "Ballistic 2-d imaging through scattering walls using an ultrafast optical kerr gate." Science (New York, N.Y.) 253, 769-771 (1991).

[6] Q. Z. Wang, X. Liang, L. Wang, P. P. Ho, and R. R. Alfano, "Fourier spatial filter acts as a temporal gate for light propagating through a turbid medium," Opt. Lett. 20, 1498-1500 (1995).

[7] E. Leith, B. Hoover, S. Grannell, K. Mills, H. Chen, and D. Dilworth, "Realization of time gating by use of spatial filtering." Applied optics 38, 1370-1376 (1999).

[8] M. Oleary, D. Boas, B. Chance, and A. Yodh, "Refraction of diffuse photon density waves," Physical Review Letters 69, 2658 (1992).
[9] L. Mullen, A. Laux, B. Concannon, E. P. Zege, I. L. Katsev, and A. S. Prikhach, "Amplitude-modulated laser imager," Applied optics 43, 3874-3892 (2004).

[10] E. Lacot, R. Day, and F. Stoeckel, "Laser optical feedback tomography," Optics letters 24, 744-746 (1999).

[11] E. Belin and V. Boucher, "An imaging system based on laser optical feedback for fog vision applications," in "SPIE Conference Series," , vol. 7088 (2008), vol. 7088, p. 70880N.

[12] J. Schmitt, A. Gandjbakhche, and R. Bonner, "Use of polarized light to discriminate short-path photons in a multiply scattering medium," Applied optics 31, 6535-6546 (1992).

[13] H. Horinaka, K. Hashimoto, K. Wada, Y. Cho, and M. Osawa, "Extraction of quasi-straightforwardpropagating photons from diffused light transmitting through a scattering medium by polarization modulation," Optics letters 20, 1501-1503 (1995).

[14] O. Emile, F. Bretenaker, and A. Floch, "Rotating polarization imaging in turbid media." Optics letters 21, 1706-1708 (1996).

[15] H. Ramachandran and A. Narayanan, "Twodimensional imaging through turbid media using a continuous wave light source," Optics Communications 154 (1998).

[16] S. Mujumdar and H. Ramachandran, "Imaging through turbid media using polarization modulation: dependence on scattering anisotropy," Optics communications 241, 1-9 (2004).

[17] J. S. Ryan and A. I. Carswell, "Laser beam broadening and depolarization in dense fogs," J. Opt. Soc. Am. 68, 900-908 (1978).

[18] M. Xu and R. Alfano, "Circular polarization memory of light," Physical Review E 72 (2005).

[19] R. Nothdurft and G. Yao, "Applying the polarization memory effect in polarization-gated subsurface imaging." Optics express 14, 4656-4661 (2006).

[20] J. A. Garland, "Some fog droplet size distributions obtained by an impaction method," Quarterly Journal of the Royal Meteorological Society 97, 483-494 (1971).

[21] M. Hayman, S. Spuler, B. Morley, and J. VanAndel, "Polarization lidar operation for measuring backscatter phase matrices of oriented scatterers," Optics express 20, 29553-29567 (2012).

[22] S. Demos and R. Alfano, "Optical polarization imaging," Applied Optics 36, 150-155 (1997).

[23] P. Terrier, V. Devlaminck, and J. Charbois, "Segmentation of rough surfaces using a polarization imaging system." Journal of the Optical Society of America. A, Optics, image science, and vision 25, 423-430 (2008).

[24] O. Morel, C. Stolz, F. Meriaudeau, and P. Gorria, "Active lighting applied to three-dimensional reconstruction of specular metallic surfaces by polariza- 
tion imaging." Applied optics 45, 4062-4068 (2006).

[25] M. Yamada, K. Ueda, I. Horiba, and N. Sugie, "Discrimination of the road condition toward understanding of vehicle driving environments," Intelligent Transportation Systems, IEEE Transactions on 2, 26-31 (2001).

[26] A. Bénière, M. Alouini, F. Goudail, and D. Dolfi, "Design and experimental validation of a snapshot polarization contrast imager." Applied optics 48, 5764-5773 (2009).

[27] H. Luo, K. Oka, D. Edward, M. Kudenov, J. Schiewgerling, and E. Dereniak, "Compact and miniature snapshot imaging polarimeter." Applied optics 47, 4413-4417 (2008).

[28] M. W. Kudenov, M. J. Escuti, N. Hagen, E. L. Dereniak, and K. Oka, "Snapshot imaging mueller matrix polarimeter using polarization gratings," Opt. Lett. 37, 1367-1369 (2012).

[29] S. Alali, T. Yang, and I. Vitkin, "Rapid time-gated polarimetric stokes imaging using photoelastic modulators." Optics letters 38, 2997-3000 (2013).

[30] V. Gruev, R. Perkins, and T. York, "Ccd polarization imaging sensor with aluminum nanowire opti- cal filters," Optics express 18, 19087-19094 (2010).

[31] M. C. Simon, "Wollaston prism with large split angle," Appl. Opt. 25, 369-376 (1986).

[32] D. Bicout, C. Brosseau, A. Martinez, and J. Schmitt, "Depolarization of multiply scattered waves by spherical diffusers: influence of the size parameter," Physical Review E 49, 1767 (1994).

[33] M. Alouini, F. Goudail, N. Roux, L. Le Hors, P. Hartemann, S. Breugnot, and D. Dolfi, "Active spectro-polarimetric imaging: signature modeling, imaging demonstrator and target detection," The European Physical Journal Applied Physics 42, 129-139 (2008).

[34] A. Bénière, F. Goudail, D. Dolfi, and M. Alouini, "Target detection in active polarization images perturbed with additive noise and illumination nonuniformity," JOSA A 26, 1678-1686 (2009).

[35] J. Fade, S. Panigrahi, and M. Alouini, "Optimal estimation in polarimetric imaging in the presence of correlated noise fluctuations," Optics Express 22, 4920-4931 (2014). 